\title{
ENTOMOLOGY
}

\section{Evaluation of a first-event sampling model for monitoring cabbage pests}

\author{
K.G. Kim, ${ }^{1}$ S. Toepfer ${ }^{2}$ \\ ${ }^{1}$ Department of Plant Protection, Pyongyang Agricultural College of Kim Il Sung University, Pyongyang, Democratic \\ People's Republic of Korea; ${ }^{2}$ CABI, Switzerland, Delémont, Switzerland
}

\begin{abstract}
First-event sampling models for monitoring diamondback moth Plutella xylostella (Lepidoptera: Plutellidae) and small white butterfly, Pieris rapae (Pieridae) are used in integrated production systems of cabbage. Decision-making accuracy and reduced labour needs of those models were unknown compared to
\end{abstract}

Correspondence: Stefan Toepfer, CABI, Rue des Grillons 1, Delémont, Switzerland.

E-mail: s.toepfer@cabi.org

Key words: Pest monitoring; operating characteristic; average sample number; simulation, integrated pest management; Plutella xylostella; Pieris rapae.

Acknowledgements: The authors are thankful to Mr Sok-Ryol Kim (Pyongyang Agricultural College of Kim Il Sung University) for help in coding the simulation processes and to CABI for training Pyongyang Agricultural College lecturers in pest monitoring and decision-making methods in integrated pest management.

Contributions: All authors have substantially contributed to conception and design, analysis and interpretation of data and results, as well as to drafting and revising the article. All approved the final version to be published. In addition to above, KGK conducted most of the model simulations.

Conflict of interest: The authors declare no potential conflict of interest.

Funding: This research was funded by Pyongyang Agricultural College of Kim Il Sung University. CABI gratefully acknowledges the core financial support from its member countries and agencies https://www.cabi.org/about-cabi/who-we-work-with/key-donors/ for full details.

Received for publication: 3 November 2020.

Accepted for publication: 3 February 2021.

${ }^{\circ}$ Copyright: the Author(s), 2021

Licensee PAGEPress, Italy

Journal of Entomological and Acarological Research 2021; 53:9448 doi:10.4081/jear.2021.9448

This article is distributed under the terms of the Creative Commons Attribution Noncommercial License (by-nc 4.0) which permits any noncommercial use, distribution, and reproduction in any medium, provided the original author(s) and source are credited. fixed-sample monitoring. This we addressed through computer simulations of the currently most used first-event sampling plan for cabbage in DPR Korea. Indeed, this sampling plan in five subplots of a cabbage field at a sampling limit of a maximum 10 plants each, appeared less labour intense than many fixed-sample monitoring plans. However, only a medium accuracy of infestation estimates and correct decision-making for or against pest control was achieved, particularly at high pest densities. If accepting such medium accuracy, the current sampling plan could be reduced from five to three subplots at a sampling limit of 10 plants each, or to a maximum of five assessed plants per each of five subplots, this is, without further loosing accuracy whilst saving labour. Such sampling requires little investment in time and might be therefore applied and validated across more cabbage productions systems of East Asia. Ultimately, first-event sampling, as other sampling plans will remain a compromise between accuracy and practicability.

\section{Introduction}

Cabbages are key vegetable crops in agricultural production of most East Asian countries (Rerkasem, 2005). Cabbages are also important vegetables in the diet of DPR Korean people (Grossrieder et al., 2005). Therefore, large areas of farming co-operatives particularly around cities, are cultivated with cabbage, particularly with Brassica oleracea var. capitata. Major cabbage pests are the caterpillars of the diamondback moth, Plutella xylostella L (Lepidoptera: Plutellidae), and of the small white butterfly, Pieris rapae L. (Lepidoptera: Pieridae) (Son and Sik, 2006).

An integrated pest management (IPM) strategy had been adopted in DPR Korea in the early 2000s to better manage cabbage pests with less input needs for synthetic pesticides (Kiefer et al., 2004). As part of this strategy, pest monitoring techniques became key components for properly deciding whether pest control measures were needed or not (Berchtold and Freuler, 1988; Hamilton et al., 2006). However, sampling techniques have to provide reasonably precise estimates of pest populations whilst remaining easy to use at a low effort of time (Berchtold and Freuler, 1988). Unfortunately, conventional pest monitoring plans of fixed sample size randomly or systematically across larger field areas are often labour intense (Bins et al., 2000; Butler and Trumble, 2012), and therefore rarely adopted by farmers.

Statisticians have proposed several ways to reduce sample sizes such as through double sampling plans (Binns and Nyrop, 1992), sequential sampling (Kuno, 1969; Binns and Nyrop, 1992; Arnaldo and Torres, 2005; Greco and Wright, 2013; Silva et al., 
2014; Pérez et al., 2015), variable intensity sampling (Hoy, 1991), binomial presence-absence sampling (Binns and Nyrop, 1992; Hamilton et al., 2006), binomial sequential sampling (Smith and Shepard, 2004; Butler and Trumble, 2012), or first-event sampling plans (Berchtold and Freuler, 1988). The accuracy as well as the time effort of either fixed sample-size plans (Hamilton et al., 2006) or sequential sampling plans (Binns and Nyrop, 1992; Arnaldo and Torres, 2005; Silva et al., 2014; Pérez et al., 2015) can be simulated through testing varying sample numbers, infestation levels, or thresholds (Binns and Nyrop, 1992; Arnaldo and Torres, 2005; Silva et al., 2014; Pérez et al., 2015) and subsequently evaluating the operating characteristic of the simulated models (Binns and Nyrop, 1992; Arnaldo and Torres, 2005; Hamilton et al., 2006; Silva AS et al., 2014).

For example, the first-event sampling used for the onion pest Liriomyza nietzkei (Diptera, Agromyzidae) estimates the proportion of leaves damaged from the positions of the first damaged leaf onwards (i.e. the first-event) and compares the estimate with the action threshold for applying a pest control measure (Berchtold and Freuler, 1988). In this sampling plan, each field is divided into 9 sample subplots and in each subplot, 10 leaves of 10 consecutive plants are examined for damage. The model for such a sampling plan for this insect revealed that this sampling technique is highly accurate in estimating damage and in determining whether the threshold is reached or not, but it appeared too labour intense.

In the early 2000s, a graphic decision-making model based on the first-event sampling approach was introduced on DPR Korean cabbage farms to better monitor lepidopteran pests (Grossrieder et al., 2005; Kim et al., 2012). This model is based on using five sample subplots across a cabbage field with a maximum of 10 monitored consecutive plants in each subplot, called the sampling limit. The sampling is stopped in each subplot once the first infested plant is found (= first event). This saves monitoring time, particularly at high pest densities. The commonly used action threshold in the country is $15 \%$ infested cabbage plants (Kim et al., 2012). Weekly samplings are usually conducted, using a graphic regression-based sampling model in relation to an action threshold line and a lower sampling threshold line. After ranking and then graphically plotting the sample results, either a control measures is applied if the sampling results are above the action threshold line, or the sampling is stopped when results are below the stop-monitoring-threshold line. Further sampling is conducted after a week if the sample results appeared between the action and lower treshold-line (Kim et al., 2012; Kuhlmann et al., 2012). Many years of applying this first-event sampling method as a decision-making tool in the integrated production of cabbage in DPR Korea led to its wide-spread adoption by farmers who understood and experienced its usefulness and practicability for monitoring caterpillar populations of $P$. xylostella and $P$. rapae. Subsequently the model was adopted in many cabbage growing areas. Farmers even demanded such decision-making tools for other pests and crops. However, despite its wide adoption, the accuracy of this decisionmaking model remained largely unknown to farmers as well as to agricultural advisers.

We therefore re-visited the first-event sampling model used for monitoring cabbage pests in DPR Korea. The objective of our study was to evaluate the currently used first-event sampling plan with regard to (i) accuracy of estimating infestation levels, (ii) accuracy of decision-making and (iii) effort in time. We simulated the effect of different numbers of sampled plants and/or assessed subplots per field at different infestation levels and thresholds (i) to simulate potential outcomes of the applied first-event samples following methods of Binns and Nyrop (1992); Bins et al. (2000); Arnaldo and Torres (2005); Silva et al. (2014) and (ii) to evaluate the operating characteristic of the model (Binns and Nyrop, 1992; Bins et al., 2000; Hamilton et al., 2006). Findings of those simulations were hoped to improve accuracy of the existing and widely applied model for pest monitoring in cabbage, and may lay the base for developing new first-event sampling approaches for other pests and/or other cabbage producing regions, ultimately saving time and efforts for farmers and agricultural advisors.

\section{Materials and Methods}

\section{First-event sampling for cabbage pests}

First-event sampling methods of pests are usually a presenceabsence sampling until the first infestation is recorded. The herestudied first-event sampling model was adapted from Hoffmeister (2004, pers. comm.), Kim et al. (2012); Kuhlmann et al. (2012). It is aimed at monitoring caterpillars of the cabbage pests diamondback moth, Plutella xylostella (Lepidoptera: Plutellidae) and the small white butterfly, Pieris rapae (Lepidoptera: Pieridae). The sampling is applied on a weekly base from one-week post-transplanting until a maximum of two weeks before harvesting depending on the pest infestation and the sampling outcome. The main cabbage crop of DPR Korea is Brassica oleracea var. capitata, wherein the first-event sampling plan is mainly applied.

Briefly, each cabbage field is divided into five subplots distributed systematically across an up to 0.5 ha field reaching good coverage, such as along a diagonal transect (Figure 1A). In every subplot, a plant is systematically chosen in a row of cabbages as a starter plant for monitoring. Random choice of the starter plant should be avoided as farmers often tend to start monitoring in the most infested hotspots of a field, leading to human bias in infestation estimates. From this plant onwards, a maximum of 10 successive plants in a row are visually examined for the pest, here caterpillars of $P$. xylostella and $P$. rapae. The criterion is a presenceabsence case, i.e. whether a plant is infested by the pest or not. The amount of damage by the pest is not assessed. This visual assessment is stopped as soon as the first plant with the pest is found among the 10 potentially to-be-sampled plants, called the 'firstevent'. The first-events occurs quickly when pest density (in other words the proportion of infested plants) is high whereas more plants may have to be checked when the pest density is low. No more than 10 plants are examined in any subplot, which is referred to as sampling limit hereafter.

The numbers of plants assessed up to the first infested plant per subplot are marked on a record sheet and subsequently given ranks from 1 to 5 in ascending order (Figure 1B). Subplots without any observed pest are given the value of infinity $(\infty)$ and are excluded in the decision-making process. If more than 2 rows of 10 plants (i.e. 2 of 5 subplots) do not yield any detection of the pest, the pest population is considered below threshold and pest monitoring is stopped for two weeks before restarting.

The graphically plotted model of the first-event sampling is used decide for or against a control measures as well as for or against further pest monitoring the following weeks (Figure 1C). The graphical model consists of, the first-event of detection at the x-axis scale $\left(x_{i}\right)$, the rank $(i)$ of the first-event on the y-axis scale, the action threshold line, and the stop-monitoring threshold line. Then, then the rank (i) of the first-events of pest detection in the five subplots are plotted against the position of the first infested plant.

The scale of the y-axis is based on the geometric distribution of ranks as follows: 
$d_{i}=-\ln (1-1 /(k+1) * i)$

[1]

where $d_{i}$ is the geometric rank of $i$ on the y-axis score, $k$ is the number of subplots and $i$ is the rank (Bins et al., 2000).

Then, the best fit to the observed data points is either visually drawn or calculated through linear regression as follows: $b=\left[\sum_{i=1}^{k}\left(d_{i} / x_{i}\right)\right] / k$

where $b$ is the slope of the regression, $i$ is the rank, $k$ is the number of subplots, $d_{i}$ the geometric rank of $i$ on the y-axis score corresponding to rank, and $x_{i}$ is the first-event number (Bins et al., 2000; Kim et al., 2012).

Step 1

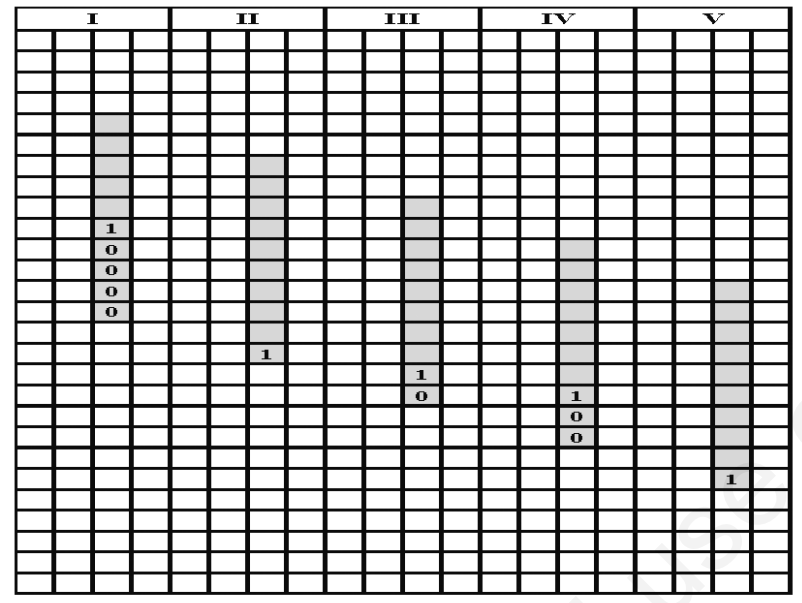

Step 2

\begin{tabular}{|l|l|l|l|l|l|}
\hline Subplot $(k$, here a row of 10 plants) & I & II & III & IV & V \\
\hline First event (First infested plant $\left.x_{i}\right)$ & 5 & 1 & 2 & 3 & 1 \\
\hline Rank $(i)$ & 5 & 1 & 3 & 4 & 2 \\
\hline
\end{tabular}

Step 3

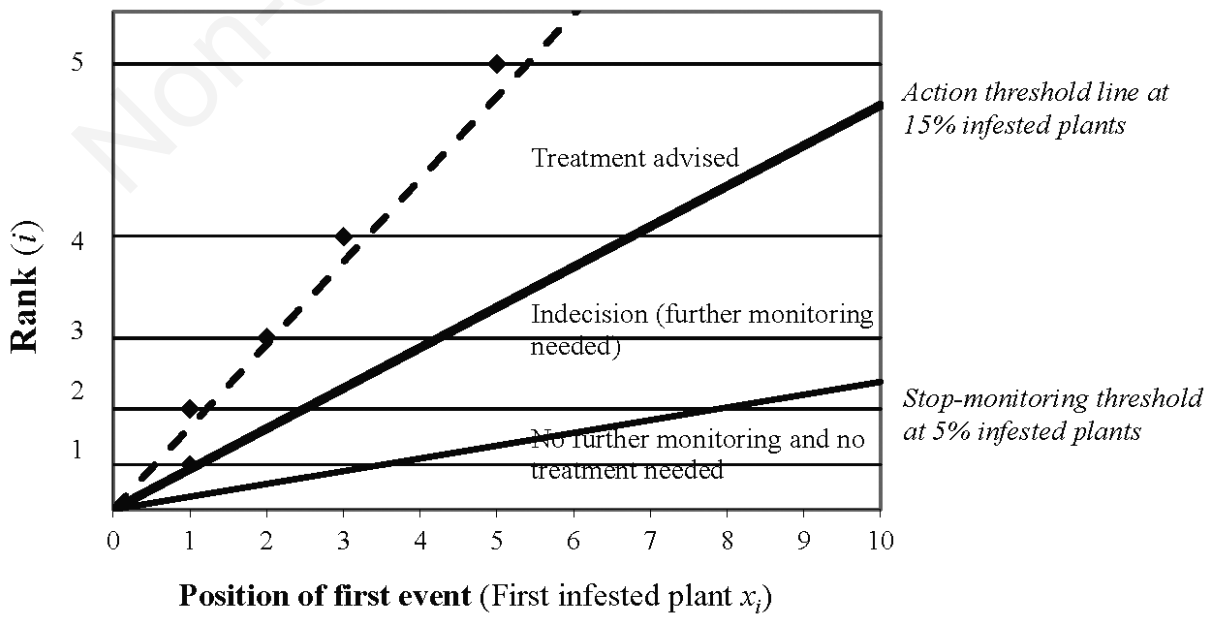

Figure 1. Example of a first-event sampling process aiding management decision for caterpillars of Plutella xylostella and Pieris rapae in cabbage adapted from Hoffmeister (2004, pers. comm.), Kim et al. (2012); Kuhlmann et al. (2012). Step 1: A cabbage field with plants (squares), sampled subplots ( $k=$ I, II, III, IV, V) of sampled rows of 10 plants (grey-shaded) as well as un-infested plants (0) and the first found infested plant (1) in each subplot. Step 2: Recording and ranking of the first events of infested plants. Step 3: Plotting ranks against numbers of the recorded first infested plants and drawing a regression line (dashed line). Upper bold line is the action threshold line at $15 \%$ infested plants above which a pest control measures is advised, and the lower line is the stop-monitoring-threshold at $\mathbf{5 \%}$ infested plants below which the infestation is so low that no action and no further monitoring is needed. 
The pest abundance is defined by the proportion of infested plants and estimated from the slope of the regression line as follows:

$p=1-\exp (-b)$

where $p$ is the estimated proportion of infested plants by pest caterpillars and $b$ is the slope of the regression line (Bins et al. 2000; Kim et al. 2012).

If the plotted line of ranks of first infested plants versus the position of the first infested plant is above the action threshold line, then a pest control measure is advised. When the line is between the action threshold and stop-monitoring threshold line, the weekly monitoring has to continue. If the line is below the stop-monitoring threshold, then monitoring can be stopped for a month and no action is needed.

\section{Evaluating accuracy of infestation estimates by first-event sampling}

The accuracy of estimates by a sampling plan is defined as the closeness of the infestation estimates to the true population density, and incorporates both bias and precision (Bins et al., 2000).

The bias of estimates was calculated as the difference between the mean of sample estimates of the proportion of infested plants $(\bar{p})$ and the true proportion of infested plants $(\mu)$ in the field from which samples are drawn:

Bias $=\bar{p}-\mu$

The precision is the closeness of an estimate to closeness to its predicted mean (Bins et al. 2000). This was here-expressed through the standard deviation (SD) of estimates of the proportion of infested plants $\left(\overline{p^{-}}\right)$as follows:
$S D=\sqrt{\sum_{i=1}^{n}(p i-\bar{p})^{2} /(n-1)}$

where $p_{i}$ is estimate of the proportion of infested plants, $\bar{p}$ is average of estimates, and $n$ is the number of sample estimates (here simulation iteration).

To evaluate whether the accuracy of pest density estimates through the widely used standard first-event sampling for cabbage pests with five sampling subplots and a 10 plants sampling limit can be improved, effects of changing subplot numbers $(k=3,5$ and 7) with a constant 10 plants sampling limit were assessed, as well as changing sampling limits $(5,10,15$ and 20 plants $)$ at a constant number of five subplots (Figure 2).

\section{Evaluating decision-making accuracy and efforts of first-event sampling}

The operating characteristic function (OC) is the probability of making a no-intervention decision against a pest, and reflected in the proportion of estimates less than the action threshold. The steeper the OC curve (= greater slope), the more sensitive the model, and the more accurate the decision-making (Bins et al., 2000; Hamilton et al., 2006). Here, the OC values of the first-event sampling method of cabbage pests were calculated as the proportion of simulations (here 100000) where the estimate of the proportion of infested plants $(\bar{p})$ was less than the action threshold.

The average sample number function (ASN) is the expected number of sampled plants required to allow a decision for or against pest control. The higher the ASN the larger the sampling efforts needed (Bins et al., 2000; Serra and Trumper, 2006; Pérez et al., 2015). Here, the sample number of a first-event sampling event is the sum of first-event numbers of subplots, and their average is the sum of the sample numbers divided by the simulation iterations (here 100000, see below).

To evaluate whether the accuracy of pest control decisions through the standard first-event sampling plan for cabbage pests

Table 1. Average probability of a correct decision and average sample numbers (ASN) required for different first-event sampling plans as well as for fixed-sample size, presence-absence, random sampling plans with an action threshold of 0.15 infestation rate of cabbage plants (based on 100,000 simulations across 0 to $100 \%$ infestation levels). Arranged from least to most accurate decision.

\begin{tabular}{|c|c|c|c|}
\hline Sampling types & Sampling plans & $\begin{array}{l}\text { Average probability } \\
\text { of correct decision }{ }^{\mathrm{a}}\end{array}$ & $\begin{array}{c}\text { Average number } \\
\text { of sampled plants required }\end{array}$ \\
\hline Presence-absence & 15 plants & 0.920 & 15 \\
\hline First-event sampling & 3 subplots, 10 plants sampling limit & 0.920 & 9.2 least effort \\
\hline First-event sampling & 5 subplots, 5 plants sampling limit & 0.922 & 11.6 \\
\hline Presence-absence & 16 plants & 0.927 & 16 \\
\hline First-event sampling & 5 subplots, 10 plants sampling limit (the currently used standard) & 0.931 & 15.3 \\
\hline First-event sampling & 5 subplots, 15 plants sampling limit & 0.931 & 17.8 \\
\hline First-event sampling & 5 subplots, 20 plants sampling limit & 0.931 & 19.8 \\
\hline Presence-absence & 17 plants & 0.933 & 17 \\
\hline Presence-absence & 18 plants & 0.938 & 18 \\
\hline First-event sampling & 7 subplots, 10 plants sampling limit & 0.938 & 21.4 \\
\hline First-event sampling & 9 subplots, 10 plants sampling limit & 0.941 & 27.5 most effort \\
\hline Presence-absence & 19 plants & 0.942 & 19 \\
\hline Presence-absence & 20 plants & 0.945 & 20 \\
\hline
\end{tabular}

aCalculated by averaging 21 values of probability of correct decisions at proportions of infested plants from 0.00 to 1.00 at an interval of 0.05 . Probability of correct decision was the operation characteristics (OC) of the applied model at proportions of infested plants less than the action thresholds and 1-OC at proportions of infested plants equal or larger than the action threshold. ${ }^{\mathrm{b}} \mathrm{Calculated}$ by averaging 21 average sample number values at proportions of infested plants from 0 to 1 at an interval of 0.05 . 
with 0.15 action threshold, 5 sampling subplots and a 10 plants sampling limit could be improved, either the action threshold ( 0.1 , 0.15 and 0.2$)$, the number of subplots ( 3,5 and 7$)$, and/or the sampling limits $(5,10,15$ and 20 plants) were varied (Figure 3$)$.

\section{Comparing first-event sampling with fixed-size presence-absence sampling}

The average probability of correct decisions and the average required sample numbers were used to compare different first-event sampling plans among each other as well as versus different fixedsize, presence-absence sampling plans (Table 1). Average probabili- ty of correct decision was calculated by averaging 21 values of probability of correct decisions at 0.00 to 1.00 proportions of infested plants at an interval of 0.05 . Average probability of correct decisions was the $\mathrm{OC}$ itself at proportions of infested plants larger than the action threshold and 1-OC at proportions of infested plants equal to or more than the action threshold. Average required sample numbers were calculated by averaging $21 \mathrm{ASN}$ values at 0.00 to 1.00 proportions of infested plants at an interval of 0.05 . The first-event sampling plans with changing number of subplots (3, 5, 7 and 9), sampling limits $(5,10,15$ and 20 plants) were compared with the fixedsize presence-absence sampling plans with sample sizes ranging
A

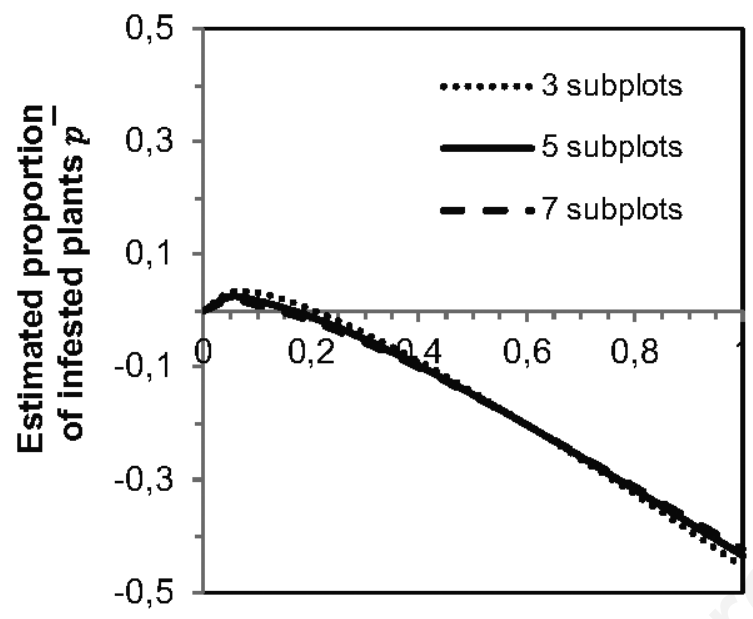

C

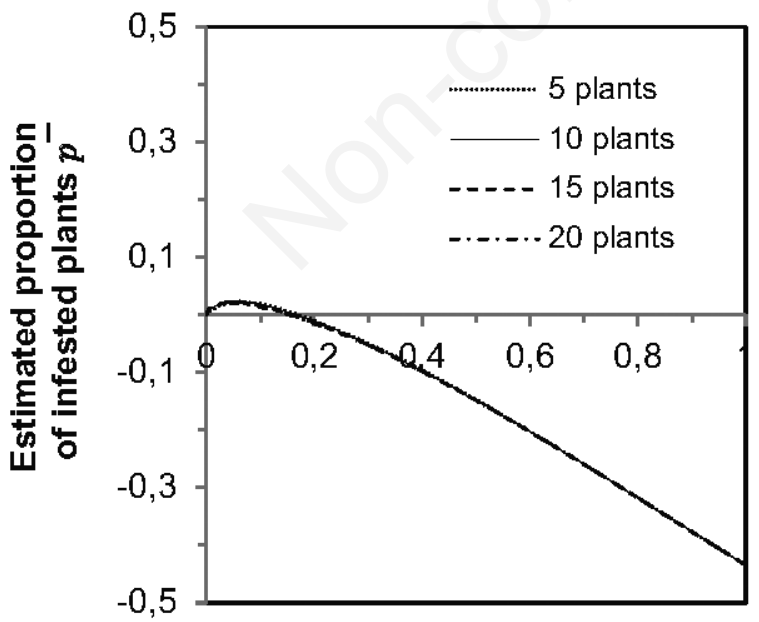

Proportion of infested plants $(\mu)$
B
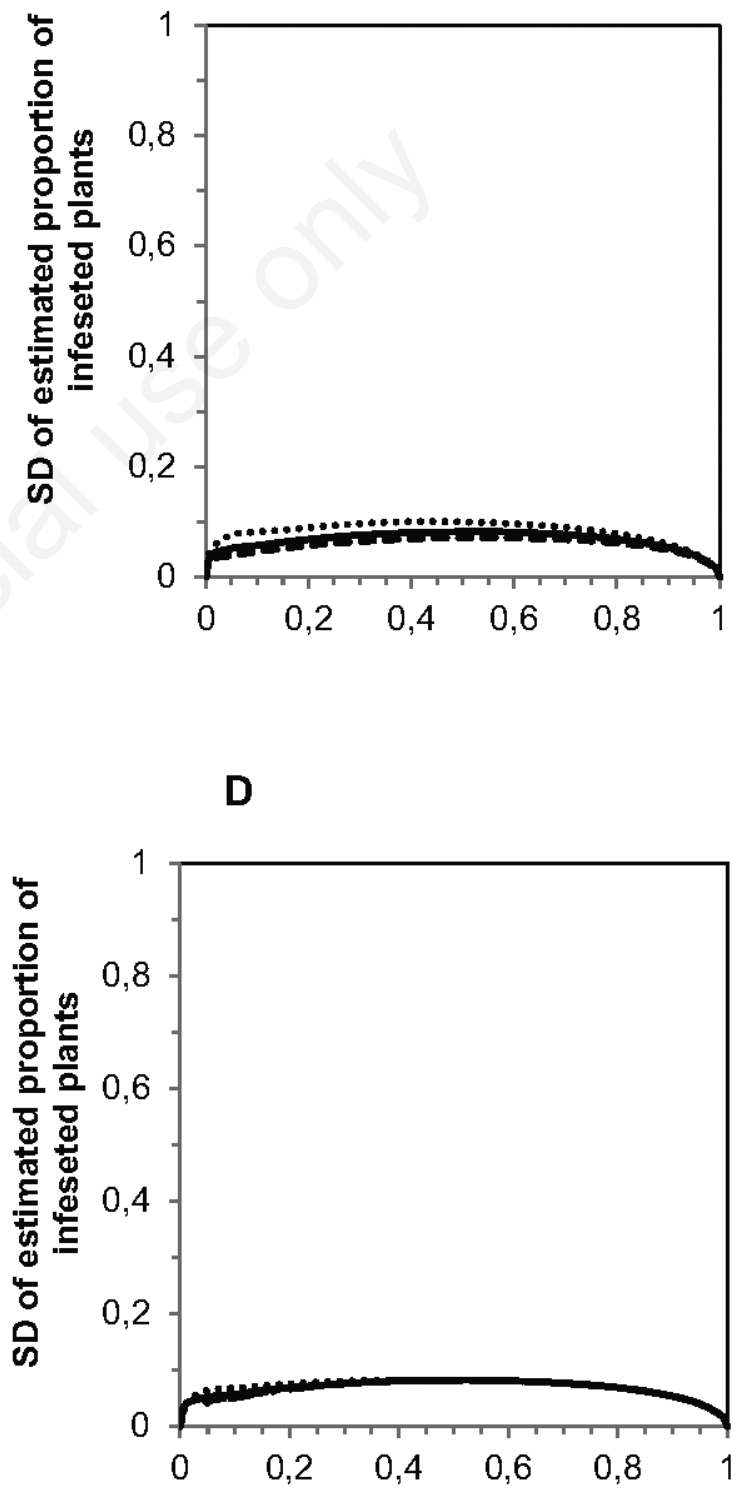

Proportion of infested plants $(\mu)$

Figure 2. Deviation of sample estimates of the proportion of infested plants $(\bar{p})$ from the true infestation of plants $(\mu)$, determined through the first-event sampling method at varying numbers of sampling subplots $(A, B)$ or varying limits of maximally assessed plants per plot (C, D). The current standard for monitoring lepidopteran cabbages pests in DPR Korea (full lines) is based on 5 subplots with 10 maximally sampled plants each. SD = standard deviation curves of estimates $(B, D) .100,000$ simulations of estimates of the proportion of infested plants $(\bar{p})$. 
from 15 to 20. A 0.15 action threshold of infested plants was used in all those simulated sampling plans.

\section{Computer simulation}

A basic assumption of the simulation was that the probability of selecting an infested plant at random in a cabbage field with a certain infestation level (proportion of infested plants $\mu$ ) would be same as the probability of getting a random number less than or equal to $\mu$ in a repeated generation of random numbers. We applied 100,000 simulations after preliminary simulations showed that this amount of simulations would provide little variation in result values. Microsoft Visual Basic 6.0 in Visual studio 6.0 Enterprise Edition (1998, Microsoft Corporation, USA) was used for coding the simulations.

\section{Computer simulation of first-event sampling}

1) Proportion of infested plants $(\mu)$ of a plant population from which samples are to be taken are defined, as well as the parameters of first-event sampling (number of subplots, sampling limit, action threshold) as described above.

2) A random number (assuming a sample plant) between 0 and 1 is generated.

3) The random number is compared with proportion of infested plants $(\mu)$. If it is more than $\mu$, it is assumed that the selected plant is not infested by a pest and the random number generation is repeated (go to 2). If it is less than or equal to $\mu$, it is assumed that the selected plant is infested by the pest and the random number generation times until then is assumed as the first-event number and then the next sampling subplot is sampled (go to 5).

4) If the times of random number generation excesses the number of maximum sampled plants per subplot (= sampling limit), random number generation is stopped and the value of infinite $(\infty)$ is given to the first-event number.

5) 2-4 steps are repeated as many as the subplot number $(k)$.

6) The first-event numbers are given ranks from 1 to $k$ in ascending order. Subplot(s) with the value of infinite $(\infty)$ are excluded in the next steps.

7) The geometric distribution of ranks for the $y$-axis is calculated using the equation [1].

8) The slope of the regression of the first-event numbers is calculated using the equation [2].

9) The estimate of the proportion of infested plants $(p)$ is calculated using the equation [3].

10) 1-9 steps are repeated as many times as the simulation iterations (here 100,000).

11) Bias and standard deviation (SD) of estimates of proportions of infested plants $(p)$ are calculated using the equations [4] and [5].

12) The operating characteristic function (OC) as the probability of making a no-intervention decision is calculated as the proportion of estimates less than the action threshold.

13) The average sample number (ASN) is calculated by dividing the sum of the first-event numbers with the simulation iterations.

1-13) steps are repeated over $\mu$ ranging from 0.00 to 1.00 at an interval of 0.05 , resulting in 21 sets of data.

\section{Computer simulation of fixed-sample presence-absence sampling}

1) Proportion of infested plants $(\mu)$ of a plant population from which samples are to be taken and sample sizes were defined.

2) Random numbers (assuming sample plants) between 0 and 1 are generated as many as the sample size. For example, in a 17 fixed-size presence-absence sampling, 17 random numbers are generated.
3) The estimate of the proportion of infested plants $(p)$ is calculated as the proportion of random numbers less than or equal to $\mu$

4) 1-3 steps are repeated as many times as the simulation iterations (here 100,000).

5) The operating characteristic function (OC) as the probability of making a no-intervention decision is calculated as the proportion of estimates less than the action threshold.

1-5) steps are repeated over $\mu$ ranging from 0.00 to 1.00 at an interval of 0.05 , resulting in 21 sets of data.

\section{Results}

\section{Accuracy of infestations estimates of the first-event sampling}

The first-event sampling plan for cabbage pests used in DPR Korea with five sampling subplots at 10 plants sampling limit each, led to a medium accuracy in estimating the proportion of infested plants at medium to low infestation levels, but to less accuracy at high infestation levels (Figure 2, full lines).

In detail, the infestation estimates $(\bar{p})$ increasingly deviated (=biased) from the real proportion of infested plants $(\mu)$ from which samples were taken with increasing infestation levels (Figure 2A,C). A slight overestimation was observed at 0.01 to 0.17 infestation rates (peak of deviation of 0.03 at $\mu=0.05$ ). From 0.2 infestation rates onwards, underestimation largely increased. Precision of estimates appeared high reflected by the flat SD curve being most un-precise (0.08) at $\mu=0.5$ (Figure 2B).

Increasing the number of sampled subplots little improved the accuracy of the first-event sampling estimates (see only slightly flattened curve), and decreasing the number of subplots little worsened accuracy (Figure 2A,B).

Similarly, changing the sampling limit little influenced the accuracy of the first-event sampling estimates (Figure 2C,D). In other words, increasing the number of sampled plants above the standard limit of 10 did not increase accuracy to any significant extent. The sampling limit might be even reduced without losing much accuracy.

\section{Decision-making accuracy and efforts of first-event sampling}

The decision-making accuracy and effort of different first-event sampling plans was more influenced by the numbers of subplots than by changing sampling limits or action thresholds. The average probability of correct decisions was high regardless whether firstevent sampling at 3 to 9 subplots of 5 to 10 plants sampling limits were applied. Increasing the number of sampled subplots and sampling limits slightly increased the accuracy of decision-making. This is reflected by steeper OC curves (i.e. the probability of making a no-intervention decision as the proportion of estimates less than the action threshold), Figure 3C,E). Obviously, the sampling effort for decision-making increased with the sampled subplots or sampling limits at decreasing infestation levels (Figure 3D,F).

The accuracy of decision-making by first-event sampling slightly decreased with increasing action threshold, reflected by less steep OC curves (Figure 3A). The sampling effort, i.e. the number of plants required to be sampled for decision-making did not change with the applied thresholds (Figure 3B). Regardless of the used thresholds, the sampling effort increased with decreasing infestation levels with a maximum of 50 plants required at close to no infestation $(\mu \sim 0)$, and a minimum of five plants needed at close to $100 \%$ infestation $(\mu \sim 1)$. 
A

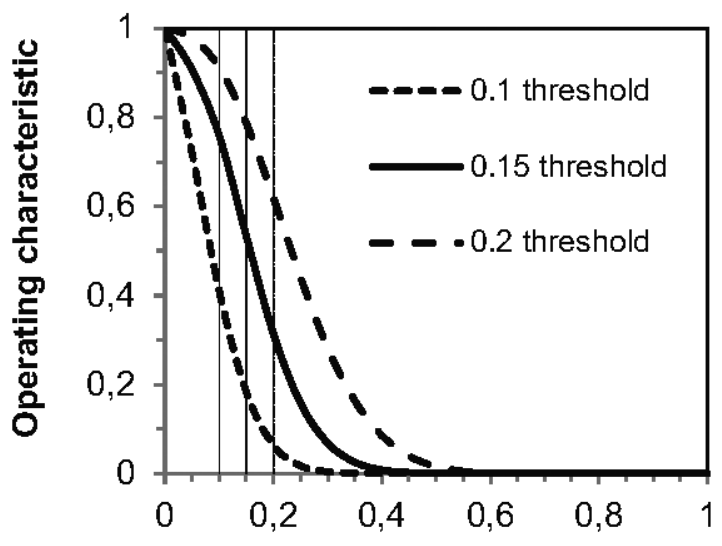

C

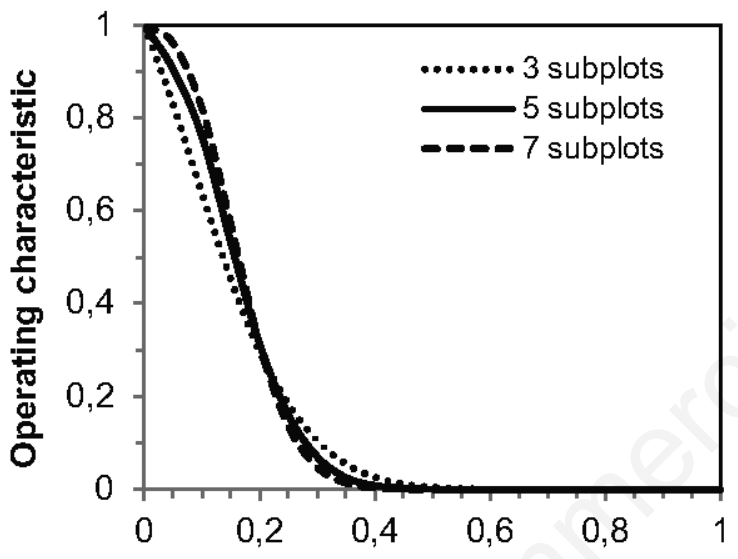

$\mathbf{E}$

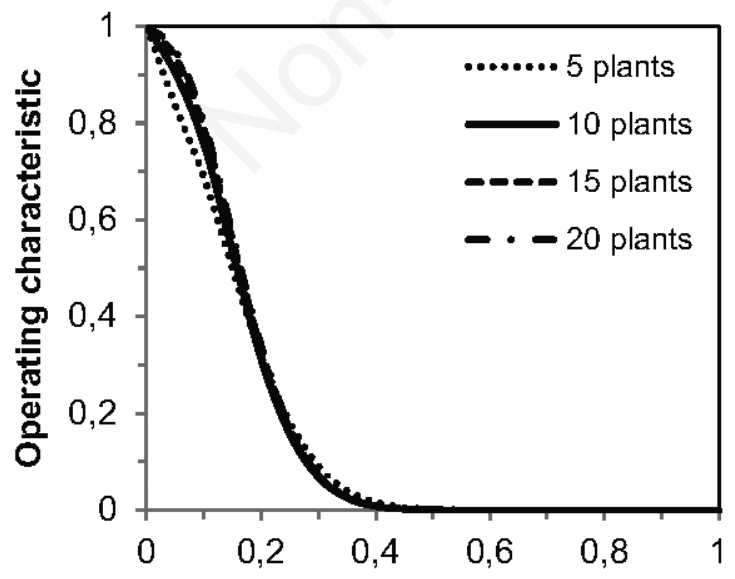

Proportion of infested plants $(\mu)$
B
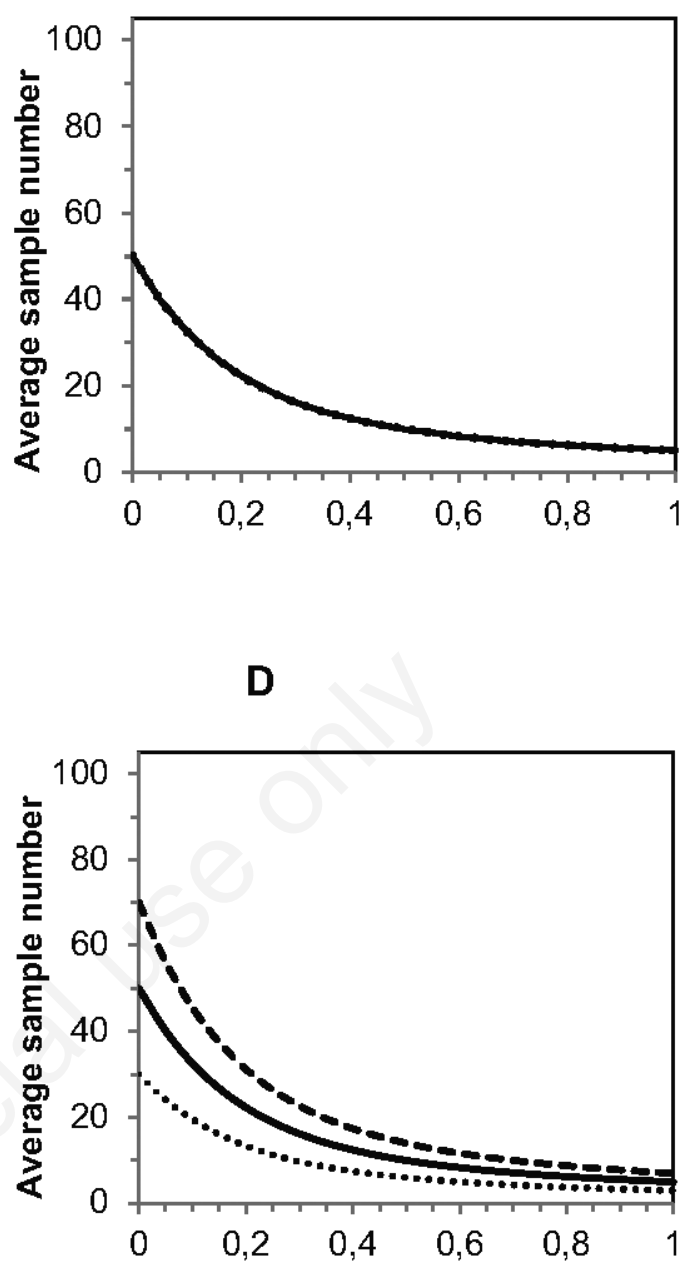

$\mathbf{F}$

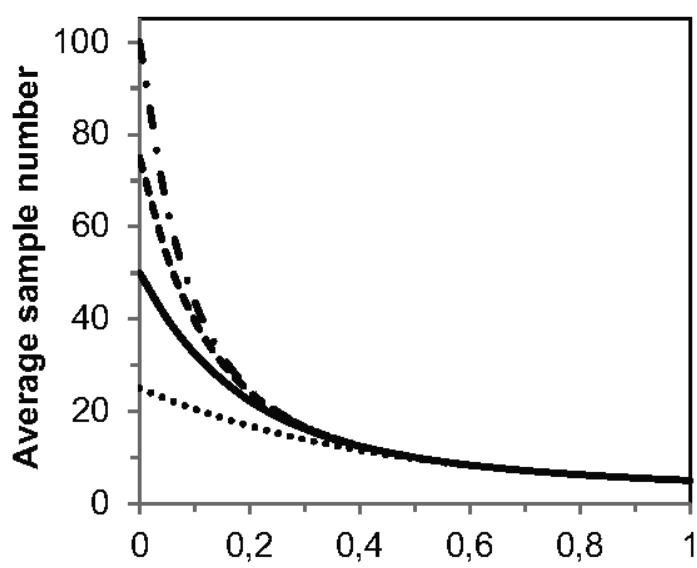

Proportion of infested plants $(\mu)$

Figure 3. Operating characteristics (OC) (A, C, E) and average sample numbers (ASN) (B, D, F) represented as model curves based on 100,000 simulations depending on the proportion of infested plants. First-event sampling plans simulated at either varying action thresholds $(0.1,0.15,0.2$ infestation rates vertical lines in fig $\mathrm{A})$, or varying numbers of samples subplots $(3,5,7$, in $\mathrm{C}, \mathrm{D})$ or varying sampling limits $(\max 5,10,15,20$ plants per plot, E, F). The current standard for monitoring lepidopteran cabbages pests in DPR Korea (full lines) is based on 5 subplots with 10 maximally sampled plants each, and at 0.15 action threshold. 


\section{Comparison between first-event sampling and fixed-sample size presence-absence sampling}

The average probability of correct decisions was comparably high with first-event sampling at three to nine subplots of five to 10 plants sampling limits as with random 15 to 20 plants presenceabsence sampling (Table. 1).

The accuracy of the first-event sampling plan currently used for cabbage pests at five subplots and a 10-plants sampling limit was comparable with the accuracy reached by the random 16 or 17 plants presence-absence sampling. The first-event sampling of maximal 10 plants on three or five subplots were less labour intense than any of the assessed fixed-sample size presence-absence sampling plans. Least sampling efforts (averaged across low to high infestation levels) was needed for the first-event sampling of maximal 10 plants in three subplots (about 9 plants sampled on average), and this at an $92 \%$ probability of a correct decision. In contrast, most sampling efforts were needed for the first-event sampling of maximal 10 plants in 9 subplots (27 to 28 plants sampled on average). The latter only reached a slightly higher accuracy than the method with least effort, i.e. 94.5 versus $92 \%$ probability.

\section{Discussion}

Farmers and agricultural extension workers are usually able to recognize caterpillars of $P$. xylostella and $P$. rapae, an obvious advantage over smaller or less distinct or mobile insect pests. Therefore, a first-event sampling model had been successfully applied for monitoring lepidopteran cabbage pests as part of the integrated pest management programmes of DPR Korea. It has been used for nearly two decades with the suggested advantages of saving labour in monitoring and decision-making and an increasing adoption rate by farmers due to its ease of use (Grossrieder et al., 2005). Due to its wide adoption over large areas of agricultural production and possible implications in case of wrong decision making, we needed to better understand the factors underlaying the accuracy of the applied first-event sampling plan. This became particularly pertinent with the increasing demands of farmers for such models for other pests and other crops.

The method of model simulations used in our study indeed helped to estimate accuracy achieved by the currently used firstevent sampling in cabbage. Bias, standard deviation, operating characteristic of no-decision probabilities, as well as numbers of required samples all appeared useful parameters as been proposed by Binns and Nyrop (1992); Bins et al. (2000); Arnaldo and Torres (2005); or Silva et al. (2014). They may also be used for evaluating other plans of first-event sampling or even presence-absence sampling, for example for other agricultural pests as well as other crops or regions. In order to develop a new model for another pest, an optimal model structure should be selected after experimentally determining the action threshold, and then balancing the effects of numbers of subplots and sampling limits on the operating characteristic of the model as well as on the numbers of samples required for accurate infestation estimates. In other words, the probability of correct or wrong decisions as well as the average required sample numbers can be used as criteria for decision-making accuracy and sampling effort in many pest-crop systems.

Our computer simulations of the standard method of first-event sampling for lepidopteran cabbage pest in DPR Korea indicated that this method can indeed be slightly less labour intense than traditional fixed-sample presence-absence monitoring plans. For example, to achieve a $92 \%$ accuracy of pest infestation estimates, about 9 plants would need to be sampled (average across different pest densities) with the first-event sampling plan and 15 plants with a fixed presence-absence sampling plan (Table 1). This is in line with many studies reporting time savings when applying sequential sampling plans over fixed sample plans (e.g. see McAuslane et al., 1987).

However, our computer simulations also revealed a medium accuracy in estimating infestation levels and consequently for decision-making. This was particularly true at high infestation levels, whilst estimates appeared relatively accurate at low or medium infestation levels (Figure 2). One may argue that such deviations of estimates may be acceptable in pest monitoring as high infestation levels are usually above the action threshold. Interestingly, changing parameters, such as sampled subplot numbers, sampling limits, or action thresholds did not much affect accuracy of the first-event sampling plan. Kuno (1969) even argue that sequential sampling may be widely adapted with fixed levels of accuracy. For example, in our study, increasing the number of sampled subplots for the first-event sampling only slightly increased its accuracy. Its sampling efforts multiplied by the number of subplots may not justify such an adaption, and would fall behind fixed-sample presence-absence sampling plans (Table 1). Similarly, the maximum number of sampled plants (sampling limit) little affected accuracy of the hereapplied first-event sampling of caterpillars of cabbage pests, and one may argue that less plants could be sampled to achieve comparable accuracy. The only medium accuracy of the analysed first-event sampling seems mainly due to its high bias of estimates, something that may be less of a problem for fixed sample-size plans. One option to improve the first-event sampling may be through using infested leaf numbers instead of infested plant numbers as the sample unit. This was, for example, applied for the onion miner Liriomyza nietzkei (Berchtold and Freuler, 1988). Another option might be to sample every second or third plant to achieve a larger the sampling coverage whilst reducing dependency of data among adjacent plants. However, this would increase sampling effort, and may hinder the wider adoption of such pest monitoring techniques at field level by farmers and extension workers.

Our analyses also showed that fixed-sample, presence-absence monitoring plans achieve comparable accuracy across different pest densities regardless of sampling 15 or up to 20 plants (Table 1). This is in contrast to Hamilton et al. (2006) who suggested a minimum of 35 plants for presence-absence sampling plans for $P$. xylostella. Nevertheless, our simulations show that different types of first-event sampling plans and fixed-sample plans can usually achieve comparably high probabilities of correct estimates $(<90 \%)$ and therefore allow correct pest management decisions.

\section{Conclusions}

In conclusion, our results indicate that the currently used firstevent sampling methods for monitoring lepidopteran cabbage pest may be further used for decision making, despite its disadvantages in relative low accuracy at high pest densities. We also showed, that the current first-event sampling plan could be reduced from currently five assessed subplots to three subplots at a sampling limit of 10 plants each, or to maximum five assessed plants per each of five subplots, without loosing much accuracy whilst saving labour. In general, first-event sampling approaches maybe easily adapted for other pest-crop combinations and regions. Ultimately, first-event sampling (as other sampling plans) remain a compromise between accuracy and practicability. 


\section{References}

ARNALDO P, TORRES L, 2005 - Spatial distribution and sampling of Thaumetopoea pityocampa (Den.\&Schiff.) (Lep.Thaumetopoeidea) populations on Pinus pinaster Ait. For. Ecol. Manage. 210: 1-7.

BERCHTOLD W, FREULER J, 1988 - First-event sampling. A new technique for estimating insect attack, using the onion miner fly, Liriomyza nietzkei, as test insect. - Entomol. Exp. Appl. 49: 251-257.

BINNS M, NYROP J, 1992 - Sampling insect populations for the purpose of IPM decision making. Annu. Rev. Entomol. 37: 427-453.

BINS M, NYROP J, WERF W VAN DER, 2000 - Sampling and monitoring in crop protection: The Theoretical Basis for Developing Practical Decision Guides. CABI Publishing, Wallingford, UK.

BUTLER C, TRUMBLE J, 2012 - Spatial dispersion and binomial sequential sampling for the potato psyllid (Hemiptera: Triozidae) on potato. - Pest Manag. Sci. 68: 865-869.

GRECO E, WRIGHT M, 2013 - Dispersion and sequential sampling plan for Xylosandrus compactus (Coleoptera: Curculionidae) infesting Hawaii coffee plantations. - Environ. Entomol. 42: 277-282.

GROSSRIEDER M, B.KIEFER, KANG S, KUHLMANN U, 2005 - Case study on knowledge transfer in cabbage IPM through farmer participatory training in DPR Korea, in: Second International Symposium on Biological Control of Arthropods. USDA Forest Service Publication FHTET-200508, Davos, Switzerland. pp 318-332.

HAMILTON A, ENDERSBY N, SCHELLHORN N, RIDLAND P, ROGERS P, JEVREMOV D, BAKER D, 2006 - Evaluation of fixed sample-size plans for Plutella xylostella (Lepidoptera: Plutellidae) on broccoli crops in Australia. - J. Econ. Entomol. 99: 2171-2176.

HOY C, 1991 - Variable-intensity sampling proportion of plants infested with pests. J. Econ. Entomol. 84: 148-157.

KIEFER B, GROSSRIEDER M, KANG SI, RIM MH, HAN CS, PYON YC, KUHLMAN U, 2004 - A farmer's manual for cabbage IPM in DPR Korea, 1st ed. CABI Europe- Switzerland and Plant Protection Institute / Academy of Agricultural Sciences, Pyongyang, DPR Korea.

KIM K, KANG S, SON K, 2012 - Some characteristics of the firstevent sampling, in: Pae, SN, Holmes, K. (Ed.), Proceedings of the Academy of Agricultural Sciences - CABI Joint Scientific Symposium on Pest Monitoring and Forecasting, Academy of Agricultural Sciences of DPR Korea and CABI, Switzerland. Academy of Agricultural sciences, Pyongyang, DPR Korea, 95-103.

KUHLMANN U, BABENDREIER D, HUNT E, GROSSRIEDER M, TOEPFER S, 2012 - Integrated Pest Management. 2nd edn. Foreign Language Books Publishing House, Pyongyang, DPR Korea.

KUNO E, 1969 - A new method of sequential sampling to obtain the population estimates with a fixed level of precision. - Res. Popul. Ecol. Kyoto 11: 127-136.

MCAUSLANE HJ, ELLIS CR, ALLEN OB, WALD A, TAYLOR LR, 1987 - Sequential sampling of adult northern and western corn rootworms (Coleoptera: Chrysomelidae) in southern Ontario. - Can. Entomol. 119: 577-585.

PÉREZ M, ISAS M, SALVATORE A, GASTAMINZA G, TRUMPER E, 2015 - Optimizing a fixed-precision sequential sampling plan for adult Acrotomopus atropunctellus (Boheman)(Coleoptera: Curculionidae), new pest on sugarcane. - Crop Prot. 74: 9-17.

RERKASEM B, 2005 - Transforming subsistence cropping in Asia. - Plant Prod. Sci. 8: 275-287.

SERRA G V, TRUMPER E V, 2006 - Sequential sampling protocols for Spodoptera frugiperda (Lepidoptera : Noctuidae), on Zea mays fields: influence of sampling unit size. - Bull. Entomol. Res. 96: 471-477.

SILVA AS, MOTA T, FERNANDES M, KASSAB S, 2014 Sequential sampling of Bemisia tuberculata (Bondar, 1923)(Hemiptera: Aleyrodidae) on cassava crop. - Ann. Brazilian Acad. Sci. 86: 889-896.

SMITH J, SHEPARD B, 2004 - A binomial sequential sampling plan using a composite threshold for caterpillar management in fresh market collard. - J. Agric. Urban Entomol. 21: 171-184.

SON C, SIK RJ, 2006 - Crop pest protection in DPR Korea. Minsitry of Agriculture, Koryo Publication Import Export Company, Pyongyang, DPR Korea. 